\title{
ARTÍCULOS
}

CRÍTICA, Revista Hispanoamericana de Filosofia

Vol. XXVIII, No. 82 (abril 1996): 3-12

\section{CARLOS E. ALCHOURRÓN BREVE BIOGRAFÍA INTELECTUAL}

\author{
GLadys PaLAU \\ Sociedad Argentina de Análisis Filosófico \\ Universidad de Buenos Aires
}

Conocí a Carlos Alchourrón hace ya demasiados años. Descubrí su capacidad intelectual en el periodo tragico argentino, en los seminarios sobre lógica modal y condicionales contrafacticos, llevados a cabo en la Sociedad Argentina de Analisis Filosófico (SADAF) y que dictaba conjuntamente con Raúl Orayen. Trabajo junto a él desde los primeros meses del gobierno democratico, como profesora adjunta de la catedra de Lógica en la Facultad de Filosofía y Letras de la Universidad de Buenos Aires. Pertenezco a su grupo de investigación desde 1987. Su muerte ocurre precisamente cuando yo estaba trabajando en mi tantas veces postergada tesis doctoral y me habia convertido en codirectora de su último proyecto de investigación. En todos los años transcurridos he sido depositaria de su saber oral, de sus borradores de trabajo, de sus publicaciones, de sus anécdotas y de los mas variados testimonios de su saber. Todo ello, mas su constante recuerdo, me ha permitido, no sin dolor, escribir estas paginas. Merecia sin duda alguna haber podido escribir su autobiografía.

El Dr. Carlos Alchourrón se graduó como abogado en el año 1957. No ejerció nunca esta profesión. En 1969 obtuvo el título de Doctor en Derecho y Ciencias Sociales con una tesis cuyo título, Clarificación lógica de algunos conceptos normativos, evidencia ya la temática central a la 
cual dedicará la mayor parte de su actividad intelectual. No obstante la magnitud de su obra, tanto en cantidad como en variedad de problemas filosóficos abordados, su principal producción científica es posible de ser analizada según tres temáticas específicas aunque estrechamente relacionadas entre sí, a saber: 1) la lógica de los sistemas normativos, 2) la lógica del cambio racional de teorías y, 3) la lógica de los condicionales "derrotables".

Las investigaciones sobre la primera temática se inician en la década de los sesenta, con el artículo "Los argumentos jurídicos a-fortiori y a pari" [1961]. La totalidad de los trabajos referidos a estos problemas, muchos de ellos escritos en colaboración con Eugenio Bulygin, fueron publicados en inglés y recientemente traducidos al español e incluidos en la compilación Análisis lógico y Derecho [1991], prologada por Georg H. von Wright. Este prominente lógico y filósofo ha desempeñado un doble papel en la vida de Alchourrón; por un lado porque sus obras fundantes en lógica deóntica son el referente ineludible de todas las investigaciones de Alchourrón y Bulygin en este campo, y por el otro porque mantendrá con él una amistad que perdurará toda su vida. No es casual que la compilación mencionada los autores la hayan dedicado al filósofo y a su esposa. En efecto, la primera contribución totalmente original en este campo la realiza Alchourrón en el artículo "Logic of Norms and Normative Propositions" [1969]; en él se muestra, por un lado, que la lógica de las normas es distinta de la lógica de las proposiciones normativas y que ello hace necesario formalizarlas en sistemas lógicos distintos, y por el otro, que el sistema propuesto por von Wright en su artículo de 1951, "Deontic Logic", es más apropiado para formalizar la lógica de las normas que la lógica de las proposiciones normativas.

No obstante la importancia de los restantes trabajos producidos bajo esta temática, los resultados fundamentales se 
plasman en el libro escrito conjuntamente con el Dr. Eugenio Bulygin, Normative Systems [1971], publicado primero en idioma inglés y traducido en 1975 al español bajo el título Introducción a la metodología de las Ciencias Juridicas y Sociales. Esta obra constituye uno de los primeros intentos de aplicar en forma sistemática la lógica deóntica al análisis de los problemas jurídicos. En efecto, en él se lleva a cabo un análisis riguroso de la noción de sistema normativo y de sus propiedades estructurales, relacionando determinados tipos de incompletitud con especies distintas de lagunas y rompiendo con la creencia tradicional de que todos los sistemas normativos eran siempre completos. El libro tuvo gran repercusión en América y Europa, siendo objeto de importantes comentarios críticos.

Sin embargo, el mencionado libro no tematizaba algunos problemas lógicos que interesaban a Alchourrón, entre ellos el de la paradoja de la eliminación, la cual aparece cuando en un sistema normativo se derogan o eliminan algunas de sus consecuencias, originándose una familia alternativa de sistemas sin criterio para elegir entre ellos y que no es atribuible ni a la consistencia ni a la completitud del sistema. Los primeros artículos que hacen referencia a esta problemática fueron escritos en colaboración también con Eugenio Bulygin y se titulan "Sobre el concepto de orden jurídico" [1976] y "Unvollstandigkeit, Widerspruchlichkeit und Umbestimmtheit der Normenordnungen" [1977], y podría decirse que constituyen la génesis de las investigaciones sobre la lógica de la teoría del cambio racional de creencias.

En efecto, el primer intento de resolver la dificultad planteada por la paradoja de la eliminación mediante una relación ordenadora entre enunciados, se expone en los artículos "Hierarchies of Regulations and Their Logics" [1981] y "Normative Order and Derogation" [1982b], el primero de ellos escrito en colaboración con David Ma- 
kinson. Este trabajo fue el primero de una serie de investigaciones realizadas sobre la lógica del cambio racional de teorías, entre las que se hace imprescindible destacar las publicadas bajo los siguientes títulos: "On the Logic of Theory Change: Contraction Functions and Their Associative Revision Functions", [1982a], "On the Logic of Theory of Change: Partial Meet Contraction and Revision Functions" [1985a] y "On the Logic of Theory Change: Safe Contraction" [1985b]. En el primero, escrito junto con David Makinson, se parte de la idea de que los problemas que se plantean con la derogación de normas en los sistemas normativos, desde un punto de vista lógico, son un caso de una dificultad más general que se presenta de manera distinta según el contexto, de ahí que el problema central sea caracterizar en forma general las funciones de revisión y contracción. Esto lleva a los autores al análisis de las consecuencias que se derivan cuando se parte de caracterizaciones distintas de dichas funciones y finalmente a postular un enfoque desde la semántica de mundos posibles. En el segundo de los trabajos, escrito en colaboración con David Makinson y Peter Gärdenfors, se incorpora la versión que de la teoría de cambios había formulado P. Gärdenfors en forma independiente y en la que los distintos tipos de cambios se caracterizan mediante postulados básicos específicos. Finalmente, se demuestra la representación de todos los postulados de la teoría de Gärdenfors, en términos de las definiciones explícitas dadas por Alchourrón y Makinson, lográndose de esta forma superar ciertas dificultades que habían quedado sin resolver en el trabajo anterior. La incorporación al grupo de Peter Gärdenfors, profesor de la Universidad de Lund, hace que de ahí en más la teoría del cambio racional de creencias se conozca con el nombre de AGM, sigla formada por las iniciales de los apellidos de sus autores. Por último, en el tercer artículo mencionado, escrito sólo con 
D. Makinson se presenta una nueva definición de la función contracción de una teoría a partir de una determinada relación entre sus elementos, probándose también cómo, en determinadas condiciones muy intuitivas respecto de una relación ordenadora particular entre los elementos de una teoría, se satisfacen los postulados de la contracción del enfoque axiomático de Gärdenfors. En un artículo posterior escrito también en colaboración con David Makinson, "Maps Between Some Different Kinds of Contraction Functions: the Finite Case" [1986a], se muestra una correspondencia entre los dos enfoques mencionados de la teoría de cambios para el caso en que la teoría esté constituida por un número finito de proposiciones no equivalentes.

AGM tuvo vastas implicaciones en distintos campos de conocimiento, en particular en la propia lógica de las proposiciones normativas y en la lógica de la llamada Inteligencia Artificial, y que trataremos de esbozar brevemente en los párrafos que siguen.

La investigación en el primer tipo de consecuencias es iniciada por el mismo Alchourrón, quién en el artículo "Conflicts of Norms and Revision of Normative Systems" [1992] analiza la problemática del cambio en los sistemas normativos y su relación con el cambio por revisión de AGM. No obstante, su aporte a la lógica de las normas culminará vinculándose con la lógica de los condicionales llamados "derrotables" en uno de los últimos artículos más importantes titulado "Philosophical Foundations of Deontic Logic and the Logic of Defeasible Conditionals" [1993]. Pero este trabajo ya forma parte de la última etapa de la obra de Alchourrón, o sea la etapa de sus contribuciones a la Inteligencia Artificial y que pasaremos de inmediato a reseñar.

Desde 1981, y en relación con el enfoque particular de H.-Neri Castañeda sobre el tema, Alchourrón estaba intere- 
sado en las complicaciones que surgían cuando se trataba de dar cuenta de la lógica de las normas condicionales. Este problema será nuevamente analizado en "Conditionality and Representation of Legal Norms" [1986b] y será objeto de un nuevo análisis vía el enfoque de los condicionales "derrotables" ${ }^{1}$ en el artículo de 1993. En él se destaca el carácter prima facie de las obligaciones jurídicas, ya dado en [1986b] y se las considera como normas condicionales "derrotables". El estudio de los condicionales derrotables se origina en las investigaciones realizadas en Inteligencia Artificial con el fin de dar cuenta de formas naturales de razonamiento diferentes de la forma de razonar deductiva y que se conoce con el nombre de razonamiento no monótono. ${ }^{2}$ En "Defeasible Logics: Demarcation and Affinities" [1994], Alchourrón crea un sistema lógico para los condicionales derrotables basado en una lógica de revisión de suposiciones en la que se introduce un operador de revisión a fin de seleccionar entre el conjunto de suposiciones, aquellas que no derroten el consecuente. Asimismo muestra que los condicionales derrotables se diferencian del condicional estricto y del condicional contrafáctico estándar en el hecho de que en ellos falla la regla del Modus Ponens. Como surge de lo dicho, el sistema propuesto por Alchourrón en este artículo, propone una caracterización de los condicionales derrotables a partir de una función de revisión

1 Intuitivamente, un enunciado condicional se dice que es un condicional "derrotable" cuando no cumple con la regla conocida con el nombre de Refuerzo del Antecedente, es decir, que el agregado de nuevos enunciados al antecedente del enunciado condicional, puede hacer "caer" o "derrotar" al consecuente y por lo tanto hacer falso al enunciado condicional.

2 Informalmente, un razonamiento se dice que es no monótono cuando no cumple con la propiedad clásica de Monotonía, es decir, cuando el agregado de nuevas premisas no preserva la corrección del razonamiento. 
análoga a la caracterizada en $\mathrm{AGM},{ }^{3}$ que posibilita conectar definitivamente la noción de revisión de la teoría del cambio racional de creencias con la noción de derrotabilidad asociada a los enunciados condicionales. Sin embargo, le quedaba aún a Alchourrón la tarea de aplicar estos resultados a la lógica deóntica y tal es lo que hace en su último artículo, aún no publicado, pero enviado a Studia Logica en noviembre de 1994, titulado "Detachment and Defeasibility in Deontic Logic". El objetivo de este artículo consiste en presentar un marco lógico en el que sea posible formalizar las obligaciones prima facie, las obligaciones condicionales derrotables, las obligaciones condicionales no derrotables y las obligaciones no derrotables y que, al mismo tiempo, permita analizar las conexiones lógicas entre los cuatro tipos de obligaciones mencionadas.

Como magistral final, su último trabajo publicado en habla hispana, "Concepciones de la lógica" [1995], está dedicado a exponer, tanto histórica como sistemáticamente, las distintas concepciones de la lógica vía la relación de consecuencia lógica adoptada. Su lectura hace evidente que la claridad y precisión de todo este trabajo sólo puede ser alcanzada por un lógico que durante cuarenta años ha estado amasando los conceptos de la lógica y ha logrado acabarlos con matiz brillante. A lo Alonzo Church en su artículo "Logic" de la Enciclopedia Británica.

3 Esto es posible porque, pese a que la noción de consecuencia lógica de AGM es la clásica, la relación específica de consecuencia del cambio por revisión es no monótona. Anteriormente ya Makinson [1991] había formulado una operación de consecuencia no-monótona mediante un debilitamiento de la propiedad de monotonía, y había traducido los postulados de revisión de AGM en términos de esta nueva operación. 


\section{BIBLIOGRAFÍA}

Alchourrón, E. Carlos, 1961, "Los argumentos jurídicos a-fortiori y a pari", revista jurídica de Buenos Aires, Buenos Aires (en ALyD, p. 3).

__ 1969, "Logic of Norms and Normative Propositions", en Logique et Analyse, Nouvel Série, September [traducción al español en ALyD, p. 25].

_-, 1971, Normative Systems, en colaboración con E. Bulygin, Springer-Verlag-Wien-New York [traducción al español, Astrea, Buenos Aires, 1972].

_, 1976 , "Sobre el concepto de orden jurídico", en colaboración con E. Bulygin, Crítica, vol. VIII, no. 23, México.

_ , 1977, "Unvollstandigkeit, Widerspruchlichkeit und Umbestimmtheit der Normenordnungen", en A. Conte, R. Hilpinen y G.H. von Wright, Deontiche Logik und Semantik, Akademische Verlagsgesellschsft.

__ 1981, "Hierarchies of Regulations and Their Logics", en colaboración con David Makinson, en Risto Hilpinen (comp.), New Studies in Deontic Logic, Norms, Actions and the Foundations of Ethics, D. Reidel.

—, 1982a, "On the Logic of Theory Change: Contraction Function and Their Associated Revision Functions", en colaboración con David Makinson, Theoria, a Swedish Journal of Philosophy, vol. XLVIII.

_, 1982 b, "Normative Order and Derogation", en A. Martino (comp.), Deontic Logic, Computational Linguistic and Legal Informatics, vol. II, North-Holland.

_ , 1985a, "On the Logic of Theory Change. Partial Meet Contraction and Revision Functions", en colaboración con David Makinson y Peter Gärdenfors, Journal of Symbolic Logics, vol. 50, no. 2.

— 1985b, "On the Logic of Theory Change: Safe Contraction", en colaboración con David Mackinson, Studia Logica, International Journal for Symbolic Logic, vol. XLIV, no. 4, (Tarski Memorial).

_ 1986a, "Maps Between Some Different Kinds of Contraction Functions: The Finite Case", en colaboración con David Makinson, Studia Logica, vol. XLV, no. 2. 
— 1986b, "Conditionality and Representation of Legal Norms", en A.A. Martino, F. Socci Natali (comps.), Automated Analysis of Legal Text, North-Holland.

__ 1991, Análisis lógico y derecho (ALyD) compilación de C.E. Alchourrón y E. Bulygin, prólogo de H. von Wright, Centro de Estudios Constitucionales, Madrid.

, 1992, "Conflicts of Norms and Revision of Normative Systems, Law and Philosophy", no. 10 [presentado en el Congreso de Miami, 1988, traducción al español en ALyD, p. 291].

— 1993, "Philosophical Foundations of Deontic Logic and the Logic of Defeasible Conditionals", en J.J.Ch. Meyer y R.J. Wieringa (comps.), Deontic Logic in Computer Science: Normative Systems Specifications, John Wiley \& Sons Ltd. —, 1994, "Defeasible Logic: Demarcation and Affinities", en G. Crocco, L. Fariñas del Cerro y A. Herzig (comps.), Conditionals and Artificial Intelligence, Oxford University Press.

, 1995, "Las concepciones de la lógica", Lógica tomo 7 en Enciclopedia Iberoamericana de Filosofía, Trotta, Consejo Superior de Investigaciones Científicas, Madrid. 


\section{SUMMARY}

This paper is a short intellectual biography of Professor Carlos E. Alchourrón. I analyze his main contributions in three fields: 1) the logic of normative systems, 2) the logic of theory change, and 3) the logic of defeasible conditionals. I review the historical development of his thought on these three subjects. Furthermore, I show how the problems that arise in the field of normative logic stimulated him to find solutions in the AGM theory and in defeasible logic. Lastly, I show how in his last papers these three fields converge in a general englobing approach. 